\section{Revista de CIENCIAS AMBIENTALES Tropical Journal of Environmental Sciences}

Revista de Ciencias Ambientales (Trop J Environ Sci) e-ISSN: 2215-3896

(Enero-Junio, 2022) . Vol 56(1): 157-177 DOI: https://doi.org/10.15359/rca.56-1.8

Open Access: www.revistas.una.ac.cr/ambientales e-mail: revista.ambientales@una.ac.cr Alvarado-Jiménez D., Herrera-Murillo J., Rojas-Marín J., González-Rodríguez M.

\title{
Propuestas de reducción de emisiones de carbono negro para fuentes móviles en Costa Rica
}

\author{
Proposals to reduce black carbon emissions for mobile sources in Costa Rica
Daniela Alvarado-Jiménez ${ }^{1}$, Jorge Herrera-Murillo², José Félix Rojas-Marín ${ }^{3}$, Manuel González-Rodríguez ${ }^{4}$

[Recibido: 13 de mayo 2021, Aceptado: 12 de octubre 2021, Corregido: 8 de noviembre 2021, Publicado: 1 de enero 2022]

\section{Resumen}

[Introducción]: Las fuentes móviles, tanto a nivel mundial como nacional, juegan un papel crítico en la generación de emisiones de carbono negro, forzador climático de vida corta que produce un gran impacto en el clima. [Objetivo]: Se proponen soluciones que permitan la mitigación de las emisiones de carbono negro, generadas por el sector transporte, dentro de un marco de viabilidad económica y técnica. [Metodología]: Se modelaron las emisiones generadas por las fuentes móviles, tomando como año base 2016, para lo cual se utilizó el software Copert 5.2. Posteriormente, se establecieron escenarios de oportunidades de reducción, los cuales fueron evaluados tomando en cuenta su potencial de disminución, la concordancia con las políticas públicas y el costo de la tecnología. [Resultados]: Las fuentes móviles emitieron 471.9 toneladas de carbono negro a la atmósfera durante el 2016, lo que equivale a 217031.7 y 707712.2 toneladas de $\mathrm{CO}_{2}$ e (tomando como potencial de calentamiento global 460 y 1500 , respectivamente). Se propone el uso de combustibles bajos en emisiones, la incorporación de vehículos eléctricos y las mejoras tecnológicas en el sistema del motor para reducir las emisiones de escape. [Conclusiones]: Las medidas de mitigación de emisiones que poseen mayor factibilidad son las relacionadas con la incorporación de combustibles bajos en emisiones, como el biodiésel y el diésel bajo en azufre. Resulta de vital importancia incluir acciones concretas para la reducción de las emisiones de carbono negro dentro de las políticas nacionales de cambio climático, pues la reducción de estas tiene impactos positivos a corto plazo.

Palabras claves: combustibles; cambio climático; contaminantes climáticos de vida corta; emisiones; mitigación.

\section{Abstract}

[Introduction]: Mobile sources, both globally and nationally, play a critical role in the generation of black carbon emissions, a short-lived climate forcer that has a great impact on the climate. [Objective]: Solutions are proposed that allow the mitigation of black carbon emissions generated by the transport sector within a framework of economic and technical feasibility. [Methodology]: The emissions generated by mobile sources were modeled, taking 2016 as the base year, for which the Copert 5.2 software was used. Subsequently, scenarios of reduction opportunities were established, which were evaluated considering their reduction potential, the concordance with public policies and the cost of the technology. [Results]: Mobile sources emitted 471.9 tons of black carbon into

1 Ingeniera en Gestión Ambiental, Escuela de Ciencias Ambientales, Universidad Nacional, Costa Rica; daniale2512@gmail.com; https://orcid.org/0000-0003-3260-3570

2 Académico, Laboratorio de Análisis Ambiental, Escuela de Ciencias Ambientales, Universidad Nacional, Costa Rica; jorge.herrera.murillo@una.ac.cr; https://orcid.org/0000-0003-4660-0178

3 Académico, Laboratorio de Análisis Ambiental, Escuela de Ciencias Ambientales, Universidad Nacional, Costa Rica; jose.rojas.marin@una.ac.cr, https://orcid.org/0000-0002-4658-3227

4 Coordinador técnico, Instituto de Normas TécnicasdeCosta Rica,Costa Rica;mgonzalez@inteco.org; https://orcid.org/0000-0001-8597-7205

(cc)




\section{Revista de CIENCIAS AMBIENTALES Tropical Journal of Environmental Sciences}

Revista de Ciencias Ambientales (Trop J Environ Sci) e-ISSN: 2215-3896

(Enero-Junio, 2022) . Vol 56(1): 157-177 DOI: https://doi.org/10.15359/rca.56-1.8

Open Access: www.revistas.una.ac.cr/ambientales e-mail: revista.ambientales@una.ac.cr Alvarado-Jiménez D., Herrera-Murillo J., Rojas-Marín J., González-Rodríguez M.

the atmosphere during 2016, which is equivalent to 217031.7 and 707712.2 tons of $\mathrm{CO}_{2} \mathrm{e}$ (taking 460 and 1500 as global warming potential, respectively). It is proposed: the use of low-emission fuels, the incorporation of electric vehicles and technological improvements in the engine system to reduce exhaust emissions. [Conclusions]: The most feasible emission mitigation measures are those related to the incorporation of low-emission fuels, such as biodiesel and low-sulfur diesel. It is vitally important to include concrete actions for the reduction of black carbon emissions within national climate change policies, since their reduction has positive impacts in the short term.

Keywords: fuels; climate change; short-lived climate pollutants; emissions; mitigation.

\section{Introducción}

El crecimiento exponencial de la población mundial, los modelos hegemónicos de consumo y la falta de conciencia a nivel ambiental han impulsado una fuerte demanda de recursos naturales. Esto ha provocado una mayor emisión de gases efecto invernadero (GEI) al ambiente, la cual es responsable del cambio climático de origen predominantemente antropogénico (Badii et al., 2015). Las emisiones de GEI han experimentado un aumento constante desde 1970; sin embargo, en la última década se ha producido el mayor incremento decenal, de acuerdo con lo señalado por el Grupo Intergubernamental de expertos sobre Cambio Climático. Sus estudios muestran que se registró un promedio anual de 1.0 giga tonelada de dióxido de carbono equivalente $\left(\mathrm{GtCO}_{2 \mathrm{e}}\right)$ entre 2000 y 2010, mientras que entre 1970 y el 2000 solo se emitieron $0.4 \mathrm{Gt}$ $\mathrm{CO}_{2 \mathrm{e}}$ por año. Asimismo, de continuar con dicho escenario, para el 2100 (según el modelo), se podrían alcanzar incrementos de temperaturas en un ámbito de $3.7^{\circ} \mathrm{C}$ a $4.8^{\circ} \mathrm{C}$ en comparación con los niveles preindustriales (IPCC, 2014). Dicha situación ha despertado, a nivel global, la necesidad de reducir estas cifras y minimizar el aumento de la temperatura media global.

Algunos gases de efecto invernadero como el $\mathrm{CO}_{2}$, son químicamente estables y persistentes en la atmósfera (de décadas a siglos), por lo que sus emisiones influyen en el clima a largo plazo. Sin embargo, existen otros agentes forzadores del calentamiento global, llamados contaminantes climáticos de vida corta (CCVC), por sus periodos menores de permanencia en la atmósfera. Entre ellos se encuentran el metano $\left(\mathrm{CH}_{4}\right)$, el óxido nitroso $\left(\mathrm{N}_{2} \mathrm{O}\right)$, el ozono $\left(\mathrm{O}_{3}\right)$ y el carbono negro $(\mathrm{CN})$. Estos agentes son responsables de un 40- $45 \%$ del calentamiento global (Ortúzar \& Tornel, 2016), por lo que una reducción en sus emisiones implicaría beneficios importantes a corto plazo en la temperatura mundial (se podría reducir hasta un $0.5^{\circ} \mathrm{C}$ del aumento previsto para el 2050).

$\mathrm{El} \mathrm{CN}$ es un componente que se encuentra principalmente en aquellas partículas con diámetro aerodinámico menor a $2.5 \mu \mathrm{m}$, conocidas como $\mathrm{PM}_{2.5}$ (Gallego Picó et al., 2012). Según indica Cruz-Núñez (2014), el CN es el mayor absorbente de radiación en forma de aerosol, siendo producido por la combustión incompleta de combustibles fósiles, biocombustibles y biomasa (UNEP y WMO, 2011). El mismo tiene un gran impacto en el clima, ya que posee un potencial de calentamiento global entre 460 y 1500 veces mayor que el $\mathrm{CO}_{2}$ (Ortúzar y Tornel, 2016), lo que lo convierte en un fuerte agente de calentamiento en el sistema climático. El CN

\begin{tabular}{|c|c|c|c|c|c|}
\hline 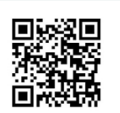 & (c) (i) () & $\underbrace{}_{\text {AMBIFNTIISS }}$ & $\begin{array}{l}\frac{9 \%}{2} \\
\frac{2}{2} \\
\text { euna }\end{array}$ & 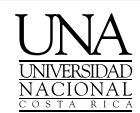 & 158 \\
\hline
\end{tabular}




\section{Revista de CIENCIAS AMBIENTALES Tropical Journal of Environmental Sciences}

Revista de Ciencias Ambientales (Trop J Environ Sci) e-ISSN: 2215-3896

(Enero-Junio, 2022) . Vol 56(1): 157-177 DOI: https://doi.org/10.15359/rca.56-1.8

Open Access: www.revistas.una.ac.cr/ambientales e-mail: revista.ambientales@una.ac.cr Alvarado-Jiménez D., Herrera-Murillo J., Rojas-Marín J., González-Rodríguez M.

genera diversos impactos climáticos que van desde variaciones en las propiedades ópticas y la vida útil de las nubes hasta modificaciones en la estabilidad y la humedad atmosféricas (Stjern et al., 2017). Además, el BC tiene otro mecanismo de fuerza cuando se deposita sobre la nieve y el hielo. Otra característica importante del CN es su tiempo de residencia en la atmósfera, el cual va de días a semanas (UNEP y WMO, 2011). Según Cruz-Núñez (2014), estaría presente en un lapso de 4 a 7 días, dependiendo de las condiciones climáticas. Algunas de las características que regulan los tiempos de residencia de este aerosol en la atmósfera y su distribución son: las cualidades microfísicas, como afinidad al agua (hidrófilo a hidrófobo); el envejecimiento de los aerosoles y la deposición húmeda; y los aspectos meteorológicos, que regulan los patrones de distribución horizontal y vertical (Fuggles et al., 2010).

Adicional a su papel en el calentamiento global, el CN también se relaciona con efectos nocivos en la salud, principalmente debido a su capacidad de penetración en el sistema respiratorio e incluso en el sistema circulatorio, lo que provoca morbilidad y mortalidad prematura (Organización Mundial de la Salud OMS, 2016). Sin embargo, aún no se ha logrado probar con certeza su toxicidad (Sims et al., 2015). Existen estudios como el de Janssen et al. (2011, citado por Sims et al., 2015), en los cuales se indica que, por unidad de concentración $\left(\mu \mathrm{gm}^{-3}\right)$, el CN puede ser más dañino que otros componentes de $\mathrm{PM}_{2.5^{\circ}}$ Otras investigaciones, como las señaladas por Gladstein et al. (2014), mencionan que este podría no ser un componente tóxico importante de las partículas finas, pero funcionaría como un portador de una amplia variedad de productos químicos de diversa toxicidad a los pulmones, las principales células de defensa del cuerpo y al sistema circulatorio.

Durante el año 2015, a nivel mundial, se emitieron aproximadamente 6.6 millones de toneladas de CN, siendo Asia, África y América Latina, las regiones que más contribuyen (UNEP, 2020). Cruz-Núñez (2014) y Sims et al. (2015) señalan que los principales emisores en los países en desarrollo son la quema de biomasa y los sólidos residenciales, mientras que fuentes como el transporte y la industria dominan en los países desarrollados.

En Costa Rica, el sector transporte juega un rol preponderante tanto en la emisión de GEI como de carbono negro. Los estudios indican que un $42.2 \%$ de la emisión total de las partículas finas $\mathrm{PM}_{2.5}$-asociadas con el $\mathrm{CN}$ - son producidas por los vehículos de carga liviana y su respectivo consumo de diesel (Programa Estado de la Nación, 2016). Así mismo, el sector transporte es el responsable del $54 \%$ de las emisiones netas de $\mathrm{CO}_{2}$ a nivel nacional (DSE-MINAE, 2016, citado por Programa Estado de la Nación, 2016).

Como parte de su agenda política en materia de cambio climático, el gobierno de Costa Rica se ha propuesto metas ambiciosas. Durante la Vigésima primera Conferencia de las Partes (COP21), el país se comprometió a una reducción de emisiones de GEI de 44 \%, comparado con un escenario Business As Usual (BAU), lo que representa una reducción de emisiones de GEI de $25 \%$, contrapuesto con las emisiones de 2012. No obstante, solo se tomó en cuenta los GEI convencionales (MINAE, 2019). Así pues, el CN quedó excluido de las políticas ante el cambio climático $\mathrm{y}$, por ende, no hay iniciativas gubernamentales para su control.

\begin{tabular}{|c|c|c|}
\hline 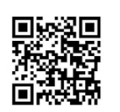 & (c) (i) () & 159 \\
\hline
\end{tabular}




\section{Revista de CIENCIAS AMBIENTALES Tropical Journal of Environmental Sciences}

Revista de Ciencias Ambientales (Trop J Environ Sci) e-ISSN: 2215-3896

(Enero-Junio, 2022) . Vol 56(1): 157-177 DOI: https://doi.org/10.15359/rca.56-1.8

Open Access: www.revistas.una.ac.cr/ambientales e-mail: revista.ambientales@una.ac.cr Alvarado-Jiménez D., Herrera-Murillo J., Rojas-Marín J., González-Rodríguez M.

El objetivo principal de la presente investigación es visualizar propuestas de reducción de emisiones de carbono negro para las fuentes móviles en Costa Rica, mediante el desarrollo de un inventario y escenarios generados a través de la modelación con el software Copert 5.2, que sirvan como herramienta de apoyo a la agenda de mitigación del cambio climático del país.

\section{Metodología}

\subsection{Elaboración del inventario de emisiones de carbono negro}

\subsubsection{Recolección y tratamiento de información}

La estimación de las emisiones, tanto de la línea base como de los escenarios de reducción, se realizó con el software COPERT que es una aplicación de Microsoft Windows ${ }^{\circledR}$ utilizada ampliamente para calcular las emisiones del sector transporte por carretera. Este permite calcular las emisiones de diferentes contaminantes (GEI, contaminantes atmosféricos y contaminantes criterio, incluyendo el CN) a diferentes escalas espaciales (nacional, regional o local) y temporales (Agencia Europea de Medio Ambiente-AEMA-, 2021). En el Cuadro 1, se muestra cada una de las fuentes de datos necesarias para la modelación de las emisiones de carbono negro, así como las limitaciones presentadas durante el proceso de gestión de la información.

Cuadro 1. Fuente, limitaciones e incertidumbre de los datos necesarios para la modelación en el software Copert 5.2 .

Table 1. Source, limitations, and uncertainty of the data used in Copert 5.2 software.

\begin{tabular}{llll}
\hline Ítem & Fuente & Descripción de la fuente y/o limitaciones & Incertidumbre \\
\hline $\begin{array}{l}\text { Población de vehículos } \\
\text { y categoría }\end{array}$ & $\begin{array}{l}\text { Base de datos del } \\
\text { Instituto Nacional }\end{array}$ & $\begin{array}{l}\text { Existen registros de vehículos hasta con 29 } \\
\text { marchamos adeudados; no obstante, para los }\end{array}$ & $0-2 \%$ \\
& de Seguros (2016). & $\begin{array}{l}\text { efectos de esta investigación, se tomó como límite } \\
\text { máximo 3 marchamos adeudados. Además, se } \\
\end{array}$ & $\begin{array}{l}\text { reclasificaron más de mil datos, según marca, } \\
\text { modelo y carrocería, debido a que (no poseían } \\
\text { una categoría específica. }\end{array}$
\end{tabular}

Población de vehículos por subcategoría
Base de datos del Instituto Nacional de Seguros (2016). Datos de pesos y medidas Datos del Consejo de Transporte Público
Se presentaron las siguientes limitaciones para la segregación de vehículos por categorías:

-Se encontraron vehículos con cilindraje incorrecto: en total, 54670 motocicletas, 129478 automóviles, 29 taxis, 22 cuadraciclos y 56040 vehículos de carga liviana. A estos datos se les asignó la moda del cilindraje de la categoría. -Se observaron incoherencias en el peso bruto y neto de vehículos pesados y autobuses, por lo que se decidió utilizar la base de datos del INS.
$0-2 \%$

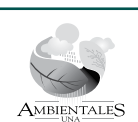




\section{Revista de CIENCIAS AMBIENTALES Tropical Journal of Environmental Sciences}

Revista de Ciencias Ambientales (Trop J Environ Sci) e-ISSN: 2215-3896

(Enero-Junio, 2022) . Vol 56(1): 157-177 DOI: https://doi.org/10.15359/rca.56-1.8

Open Access: www.revistas.una.ac.cr/ambientales e-mail: revista.ambientales@una.ac.cr Alvarado-Jiménez D., Herrera-Murillo J., Rojas-Marín J., González-Rodríguez M.

\begin{tabular}{|c|c|c|c|}
\hline Ítem & Fuente & Descripción de la fuente y/o limitaciones & Incertidumbre \\
\hline $\begin{array}{l}\text { Población de vehículos } \\
\text { por tecnología }\end{array}$ & $\begin{array}{l}\text { Base de datos del } \\
\text { Instituto Nacional } \\
\text { de Seguros (2016). }\end{array}$ & $\begin{array}{l}\text { Se establece que la tecnología de los vehículos está } \\
\text { normada según el año de fabricación, de acuerdo } \\
\text { con los estándares internacionales }\end{array}$ & $0-2 \%$ \\
\hline Kilometraje anual & $\begin{array}{l}\text { Encuesta de } \\
\text { Consumo } \\
\text { Energético Nacional } \\
\text { en el Sector } \\
\text { Transporte (MOPT, } \\
\text { 2013). }\end{array}$ & $\begin{array}{l}\text { Se toma el dato teórico del recorrido promedio } \\
\text { semanal. }\end{array}$ & $0-2 \%$ \\
\hline $\begin{array}{l}\text { Rural share, highway } \\
\text { share y urban share. }\end{array}$ & Copert 5.2 & $\begin{array}{l}\text { No se obtienen datos del kilometraje de cada } \\
\text { tecnología por modo de conducción. Se utilizan } \\
\text { las entradas por defecto del software. }\end{array}$ & $30-100 \%$ \\
\hline Velocidad urbana & $\begin{array}{l}\text { Datos obtenidos } \\
\text { del Ministerio de } \\
\text { Obras Públicas y } \\
\text { Transportes-Waze }\end{array}$ & $\begin{array}{l}\text { Se contemplan dentro de esta categoría las rutas } \\
\text { de tipo } 2 \text { (rutas secundarias que comunican } \\
\text { cabeceras de provincia). El dato de velocidad } \\
\text { promedio se toma de } 51 \text { de estas rutas. }\end{array}$ & $0-2 \%$ \\
\hline Velocidad autopista & $\begin{array}{l}\text { Datos obtenidos } \\
\text { del Ministerio de } \\
\text { Obras Públicas y } \\
\text { Transportes-Waze }\end{array}$ & $\begin{array}{l}\text { Se contemplan dentro de esta categoría las rutas } \\
\text { de tipo } 1 \text { (rutas primarias que comunican la } \\
\text { capital con las otras provincias o con las fronteras. } \\
\text { El dato de velocidad promedio se toma de } 9 \text { de } \\
\text { estas rutas (rutas: } 1,2,3,5,10,22,27,32 \text { y } 39 \text { ). }\end{array}$ & $0-2 \%$ \\
\hline Velocidad rural & $\begin{array}{l}\text { Dato obtenido del } \\
\text { Plan de Transporte } \\
\text { 2011-2035 }\end{array}$ & $\begin{array}{l}\text { No se encontraron datos de las velocidades en } \\
\text { carreteras rurales, por lo que se utilizó un valor } \\
\text { teórico. }\end{array}$ & $0-2 \%$ \\
\hline $\begin{array}{l}\text { Promedio de } \\
\text { temperatura mínima } \\
\text { mensual }\end{array}$ & $\begin{array}{l}\text { Dato del Instituto } \\
\text { Meteorológico } \\
\text { Nacional }\end{array}$ & $\begin{array}{l}\text { El dato utilizado se obtuvo a partir de la } \\
\text { temperatura mínima promedio de } 46 \text { estaciones } \\
\text { meteorológicas ubicadas en todo el país. }\end{array}$ & $0-2 \%$ \\
\hline $\begin{array}{l}\text { Promedio de la } \\
\text { temperatura máxima } \\
\text { mensual }\end{array}$ & $\begin{array}{l}\text { Dato del Instituto } \\
\text { Meteorológico } \\
\text { Nacional }\end{array}$ & $\begin{array}{l}\text { El dato utilizado se obtuvo a partir de la } \\
\text { temperatura máxima promedio de } 46 \text { estaciones } \\
\text { meteorológicas ubicadas en todo el país. }\end{array}$ & $0-2 \%$ \\
\hline Humedad relativa (\%) & $\begin{array}{l}\text { Dato del Instituto } \\
\text { Meteorológico } \\
\text { Nacional }\end{array}$ & $\begin{array}{l}\text { El dato utilizado se obtuvo a partir del porcentaje } \\
\text { de humedad promedio de } 30 \text { estaciones } \\
\text { meteorológicas ubicadas en todo el país. }\end{array}$ & $0-2 \%$ \\
\hline $\begin{array}{l}\text { Kilometraje de la flota } \\
\text { vehicular }\end{array}$ & Copert 5.2 & $\begin{array}{l}\text { No se contó con datos referentes al kilometraje de } \\
\text { los vehículos. }\end{array}$ & $30-100 \%$ \\
\hline $\begin{array}{l}\text { Especificaciones de } \\
\text { combustible (H:C } \\
\text { relación, O:C relación). }\end{array}$ & Copert 5.2 & $\begin{array}{l}\text { No se contó con datos relacionados a las } \\
\text { especificaciones de relación H:C y O:C. Se utilizan } \\
\text { datos del Copert } 5.2\end{array}$ & $30-100 \%$ \\
\hline
\end{tabular}

(c)




\section{Revista de CIENCIAS AMBIENTALES Tropical Journal of Environmental Sciences}

Revista de Ciencias Ambientales (Trop J Environ Sci) e-ISSN: 2215-3896

(Enero-Junio, 2022) . Vol 56(1): 157-177 DOI: https://doi.org/10.15359/rca.56-1.8

Open Access: www.revistas.una.ac.cr/ambientales e-mail: revista.ambientales@una.ac.cr Alvarado-Jiménez D., Herrera-Murillo J., Rojas-Marín J.,

González-Rodríguez M

\begin{tabular}{llll}
\hline Ítem & Fuente & Descripción de la fuente y/o limitaciones & Incertidumbre \\
\hline $\begin{array}{l}\text { Especificaciones de las } \\
\text { especies contenidas en } \\
\text { el combustible. }\end{array}$ & $\begin{array}{l}\text { Datos de Refinadora } \\
\text { Costarricense de } \\
\text { Petróleo y Copert } \\
5.2 .\end{array}$ & $\begin{array}{l}\text { Se utilizaron los datos de la concentración de } \\
\text { azufre y componentes oxigenados presentes en los } \\
\text { combustibles. }\end{array}$ & $30-100 \%$ \\
$\begin{array}{l}\text { Contenido de } \\
\text { lubricante y } \\
\text { especificaciones. }\end{array}$ & Copert 5.2 & $\begin{array}{l}\text { No se contó con datos relacionados a las } \\
\text { especificaciones de los lubricantes utilizados. Se } \\
\text { utilizan parámetros del Copert 5.2. }\end{array}$ & $30-100 \%$ \\
$\begin{array}{l}\text { Venta total de } \\
\text { combustibles. }\end{array}$ & $\begin{array}{l}\text { Balance de Energía } \\
2016, \text { MINAE, }\end{array}$ & $\begin{array}{l}\text { Únicamente se tomó en cuenta el consumo de } \\
\text { gasolina y diésel, pues la población de vehículos } \\
\text { híbridos o que utilicen otra fuente de combustible } \\
\text { como GLP rondan el 0.1\% de la flota vehicular. }\end{array}$ & $0-2 \%$ \\
$\begin{array}{l}\text { Dirección de } \\
\text { Energía. } \\
\text { por tipo de vehículo. }\end{array}$ & Copert 5.2 & $\begin{array}{l}\text { Factores de emisión internacionales } \\
\text { suministrados por el software Copert 5.2. }\end{array}$ & $30-100 \%$ \\
\hline
\end{tabular}

\subsubsection{Procesamiento de información mediante el modelo computacional Copert 5.2 y cálculo de emisiones del sector transporte \\ En el Cuadro 2 y Cuadro 3, se detallan los datos de entrada para la modelación del Copert}

5.2 .

Cuadro 2. Promedios mensuales de temperatura (máximas y mínimas) y humedades registradas en Costa Rica durante el 2016.

Table 2. Monthly averages of temperature (maximum and minimum) and humidity registered in Costa Rica during 2016.

\begin{tabular}{llll}
\hline Mes & Temperatura Mín $\left({ }^{\circ} \mathrm{C}\right)$ & Temperatura Máx $\left({ }^{\circ} \mathrm{C}\right)$ & Humedad $(\%)$ \\
\hline Enero & 18.63 & 28.79 & 79.5 \\
Febrero & 18.68 & 28.56 & 77.6 \\
Marzo & 19.58 & 30.38 & 76.8 \\
Abril & 20.08 & 30.49 & 77.3 \\
Mayo & 22.06 & 29.46 & 82.8 \\
Junio & 19.27 & 27.74 & 86.9 \\
Julio & 19.54 & 27.63 & 87.2 \\
Agosto & 19.62 & 32.25 & 86.1 \\
Septiembre & 19.59 & 28.50 & 86.5 \\
Octubre & 19.14 & 27.96 & 87.6 \\
Noviembre & 18.47 & 25.21 & 88.2 \\
Diciembre & 18.87 & 26.96 & 87.1 \\
\hline
\end{tabular}

Fuente: IMN (2017).

(cc)




\section{Revista de CIENCIAS AMBIENTALES Tropical Journal of Environmental Sciences}

Revista de Ciencias Ambientales (Trop J Environ Sci) e-ISSN: 2215-3896

(Enero-Junio, 2022) . Vol 56(1): 157-177 DOI: https://doi.org/10.15359/rca.56-1.8

Open Access: www.revistas.una.ac.cr/ambientales e-mail: revista.ambientales@una.ac.cr Alvarado-Jiménez D., Herrera-Murillo J., Rojas-Marín J., González-Rodríguez M

Cuadro 3. Datos de actividad registrados en el año 2016 utilizados en la corrida del modelo Table 3. Activity data registered in 2016 used in the model run

\begin{tabular}{lc}
\hline Variable & Datos de actividad \\
\hline Consumo de diésel $(\mathrm{TJ}) \mathrm{a}$ & 35871 \\
Consumo de gasolina regular $(\mathrm{TJ})^{\mathrm{a}}$ & 20131 \\
Consumo de gasolina súper $(\mathrm{TJ})^{\mathrm{a}}$ & 20173 \\
Velocidad media en autopista $(\mathrm{km} / \mathrm{h})^{\mathrm{b}}$ & 51.6 \\
Velocidad media en zona urbana $(\mathrm{km} / \mathrm{h})^{\mathrm{b}}$ & 35.3 \\
Velocidad media en zona rural $(\mathrm{km} / \mathrm{h})^{\mathrm{c}}$ & 35 \\
\hline
\end{tabular}

a Dato tomado del Balance Energético de Costa Rica (2016).

b Promedio de las rutas de las cuales tiene registro el MOPT.

${ }^{c}$ Dato tomado del Plan Nacional de Transportes de Costa Rica 2011-2035.

En total se clasificaron 1459555 vehículos, de acuerdo con el tipo de placa (particular o de pasajeros, carga liviana, carga pesada, cuadraciclos y motocicletas), el peso bruto o el cilindraje y el estándar de emisiones por año del modelo. Una vez que se ingresaron los datos de entrada al software, este calcula las emisiones mediante un enfoque de tipología "top-down". El software estima el total de emisiones combinando información técnica específica (ej. factores de emisión) y los datos de actividad de la flota de transporte (ej. total de kilómetros recorridos). Asimismo, como indica Ceballos Marcillo (2016), se toma en cuenta los siguientes parámetros:

- $\quad$ Tipo de combustible (gasolinas, diésel, gas natural comprimido, biodiésel)

- Forma estructural (categoría y subcategoría)

- Tipo de tecnología de control de emisiones (convencional, Euro I, II, III, IV, V, VI)

- Formulación de patrones de conducción dependiente de la velocidad: conducción urbana, conducción rural y conducción en autopista.

Una vez introducidas esas variables, el Copert 5.2 realiza el cálculo final mediante la suma de 3 fuentes distintas de emisiones: aquellas obtenidas cuando el motor se encuentra en una fase térmica estabilizada (hot emissions), las recabadas durante la fase de calentamiento del sistema (cold emissions) y, finalmente, las obtenidas por evaporación del combustible (fuel evaporative emissions), según se indica en la Ecuación 1:

$$
E T O T A L=E H O T+E C O L D+E E V A
$$

\begin{tabular}{|c|c|c|c|c|c|}
\hline 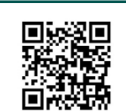 & (c) () $(0)$ & $\theta_{\text {AMEIENTIIES }}$ & $\frac{O \%}{2 \%}$ & $\frac{\text { UNA }}{\frac{\text { UNIVERSIDAD }}{\text { NACIONAL }}}$ & 163 \\
\hline
\end{tabular}




\section{Revista de CIENCIAS AMBIENTALES Tropical Journal of Environmental Sciences}

Revista de Ciencias Ambientales (Trop J Environ Sci) e-ISSN: 2215-3896

(Enero-Junio, 2022) . Vol 56(1): 157-177 DOI: https://doi.org/10.15359/rca.56-1.8

Open Access: www.revistas.una.ac.cr/ambientales e-mail: revista.ambientales@una.ac.cr Alvarado-Jiménez D., Herrera-Murillo J., Rojas-Marín J., González-Rodríguez M.

donde $\mathrm{E}_{\text {TотаL }}$ se refiere a las emisiones totales de $\mathrm{CN}$; $\mathrm{E}_{\text {HOт }}$ y $\mathrm{E}_{\mathrm{COLD}}$ son las emisiones en caliente y en frío, $\mathrm{E}_{\mathrm{VVA}}$ son las emisiones evaporativas. En el caso del $\mathrm{CN}$, únicamente se incluyen las emisiones en caliente, pues, por su naturaleza, las emisiones se producen durante el proceso de combustión, por lo que no se registran las $\mathrm{E}_{\mathrm{VVA}}$. En cuanto a las $\mathrm{E}_{\mathrm{COLD}}$, el software no contabiliza por default este contaminante (Ceballos Marcillo, 2016). Con los resultados obtenidos de las emisiones de carbono negro, se procedió a calcular las emisiones de $\mathrm{CO}_{2 \mathrm{e}}$ con un potencial de calentamiento global (PCG). Para esta actividad, se utilizó la Ecuación 2:

$$
E C O_{2 e}=E_{C N} x P C G
$$

donde $\mathrm{ECO}_{2 \mathrm{e}}$ se refiere a las emisiones de $\mathrm{CO}_{2 \mathrm{e}}$ calculadas; $\mathrm{E}_{\mathrm{CN}}$ es la emisión de carbono negro para determinado periodo de tiempo, y $\mathrm{PCG}_{\mathrm{CN}}$ aduce al potencial de calentamiento global del carbono negro. Para este estudio se utilizaron dos potenciales de calentamiento global: 460 y 1500 (UNEP, 2020), debido a que aún las investigaciones científicas no han llegado a un consenso de un valor puntual.

\subsubsection{Cálculo de incertidumbre}

Para el cálculo de la incertidumbre, se utilizó la metodología de la Guía de Inventarios de Emisiones de Contaminantes Atmosféricos de la Agencia Europea de Medio Ambiente (2021). En esta se realiza una descripción cualitativa de la incertidumbre de los datos de actividad y los factores del inventario de emisiones. Esta metodología es similar a la utilizada para el cálculo de incertidumbre de los contaminantes tóxicos del aire para Costa Rica. Dicha evaluación se muestra en el Cuadro 1.

\subsubsection{Establecimiento de medidas de reducción del sector}

\subsubsection{Identificación, evaluación y divulgación de medidas de reducción}

Mediante la revisión bibliográfica, se identificaron posibles medidas de reducción de emisiones de carbono negro para la flotilla vehicular costarricense. Adicionalmente, se realizó una evaluación de estas para asegurar su factibilidad y pertinencia. Para ello, primeramente, se elaboró una matriz que contempló aspectos como: estimación de potencial de reducción (toneladas de carbono negro) para dos escenarios 2025 y 2030, concordancia con las políticas públicas y costo de la tecnología. Finalmente, cada medida fue evaluada mediante un proceso de suma, de acuerdo con los criterios establecidos en el Cuadro 4, con el objetivo de hacer propuestas acordes con el contexto costarricense.

\begin{tabular}{|c|c|c|c|c|c|}
\hline 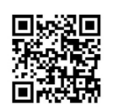 & (c) () () () & $\underset{\text { AMBIENALILS }}{3}$ & $\frac{1 \%}{2 \%}$ & $\frac{\text { UNA }}{\frac{\text { UNIEESIDAD }}{\frac{N A C I O N A L}{N A S T A R A L}}}$ & 164 \\
\hline
\end{tabular}




\section{Revista de CIENCIAS AMBIENTALES Tropical Journal of Environmental Sciences}

(Enero-Junio, 2022) . Vol 56(1): 157-177 DOI: https://doi.org/10.15359/rca.56-1.8

Open Access: www.revistas.una.ac.cr/ambientales e-mail: revista.ambientales@una.ac.cr Alvarado-Jiménez D., Herrera-Murillo J., Rojas-Marín J., González-Rodríguez M.

Cuadro 4. Criterios utilizados para la evaluación de las medidas de reducción de carbono negro.

Table 4. Criteria used for the evaluation of black carbon reduction measures.

\begin{tabular}{|c|c|c|c|c|c|c|}
\hline Escala & $\begin{array}{l}\text { Concordancia con } \\
\text { políticas públicas }\end{array}$ & Puntuación & $\begin{array}{l}\text { Costo de la } \\
\text { tecnología }\end{array}$ & Puntuación & $\begin{array}{l}\text { Potencial de } \\
\text { reducción }\end{array}$ & Puntuación \\
\hline Alto & $\begin{array}{l}\text { Existen políticas } \\
\text { públicas o legislación } \\
\text { que respalden } \\
\text { directamente la } \\
\text { medida de reducción. }\end{array}$ & 3 & $\begin{array}{l}\text { Costo de la } \\
\text { tecnología implica } \\
\text { un incremento } \\
\text { en el precio del } \\
\text { servicio; vehículo } \\
\text { de un } 5 \% \text { o } \\
\text { más comparado } \\
\text { con tecnologías } \\
\text { utilizadas } \\
\text { actualmente. }\end{array}$ & 1 & $\begin{array}{l}\text { Reducción } \\
\text { mayor a un } \\
10 \%\end{array}$ & 3 \\
\hline Medio & $\begin{array}{l}\text { Existen políticas } \\
\text { públicas o legislación } \\
\text { vinculada con la } \\
\text { medida de reducción; } \\
\text { sin embargo, esta } \\
\text { no se plantea } \\
\text { explícitamente. }\end{array}$ & 2 & $\begin{array}{l}\text { Costo de la } \\
\text { tecnología implica } \\
\text { un incremento } \\
\text { en el precio del } \\
\text { servicio; vehículo } \\
\text { entre un } 0.1 \%-5 \\
\% \text { comparado } \\
\text { con tecnologías } \\
\text { utilizadas } \\
\text { actualmente. }\end{array}$ & 2 & $\begin{array}{l}\text { Reducción de } \\
\text { un } 5 \text { a un } 10 \%\end{array}$ & 2 \\
\hline Bajo & $\begin{array}{l}\text { No existen políticas } \\
\text { públicas o legislación } \\
\text { vinculada con la } \\
\text { medida de reducción. }\end{array}$ & 1 & $\begin{array}{l}\text { Costo de la } \\
\text { tecnología no } \\
\text { implica un } \\
\text { incremento en el } \\
\text { precio del servicio. }\end{array}$ & 3 & $\begin{array}{l}\text { Reducción } \\
\text { menor a un } \\
5 \%\end{array}$ & 1 \\
\hline
\end{tabular}

\section{Resultados y discusión}

\subsection{Emisiones del sector transporte}

En total se contabilizan 471.9 toneladas de carbono negro emitidas a la atmósfera por la flotilla vehicular durante el 2016, con un error medio estimado del $8.7 \%$. Dicho resultado es un valor intermedio respecto de los obtenidos en investigaciones similares reportadas en los inventarios de carbono negro de México y los Estados Unidos. Visualizándose que en Costa Rica se produce un $48 \%$ menos de emisiones por vehículo (ton/vehículo) con respecto a México, pero un $25 \%$ más que en los Estados Unidos (Cuadro 5). Estas diferencias pueden deberse a factores como la edad de la flotilla vehicular, mayor presencia de vehículos en las categorías con altos factores de emisión y las características del combustible.

\begin{tabular}{|c|c|c|}
\hline 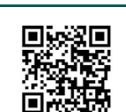 & (c) (i) (5) (2) & 165 \\
\hline
\end{tabular}




\section{Revista de CIENCIAS AMBIENTALES Tropical Journal of Environmental Sciences}

Revista de Ciencias Ambientales (Trop J Environ Sci) e-ISSN: 2215-3896

(Enero-Junio, 2022) . Vol 56(1): 157-177 DOI: https://doi.org/10.15359/rca.56-1.8 Open Access: www.revistas.una.ac.cr/ambientales e-mail: revista.ambientales@una.ac.cr Alvarado-Jiménez D., Herrera-Murillo J., Rojas-Marín J., González-Rodríguez M.

Cuadro 5. Comparación de las emisiones de carbono negro registradas para 3 países.

Table 5. Comparison of recorded black carbon emissions for three countries.

\begin{tabular}{lllllll}
\hline Inventario & Metodología & $\begin{array}{l}\text { Cantidad de } \\
\text { vehículos }\end{array}$ & $\begin{array}{l}\text { Emisiones } \\
\text { CN (Mg) }\end{array}$ & Año & $\begin{array}{l}\text { Emisiones (ton/ } \\
\text { vehículo) }\end{array}$ & Fuente \\
\hline México & top-down & 22138478 & 13870 & 2005 & 0.00062651 & $\begin{array}{l}\text { Cruz-Núñez } \\
(2014)\end{array}$ \\
USA & top-down & 247421120 & 63600 & 2005 & 0.00025705 & Chow et al. (2011) \\
Costa Rica & top-down & 1459555 & 471.9 & 2016 & 0.00032325 & -- \\
\hline
\end{tabular}

Además, en la Figura 1 se muestran las emisiones de carbono negro por categoría de vehículo: el 86.6 \% de estas emisiones se producen por 3 categorías: vehículos de carga pesada, vehículos de carga liviana con motor diésel y, en menor proporción, los autobuses. Estos resultados son similares a los obtenidos en las emisiones por tipo de combustible: el $96.9 \%$ proviene de vehículos de encendido por compresión (vehículos diésel); y únicamente un $3.1 \%$ es producido por aquellos con motor a base de gasolina. Al respecto, Cruz-Núñez (2014) señala que el $83 \%$ de las emisiones de carbono negro provienen de buses y vehículos de carga, tanto liviana como pesada. Asimismo, Chow et al. (2011) señalan que las emisiones en los motores diésel pueden ser de 20 a 40 \% mayores que las de un motor gasolina, debido a la ineficiencia en la combustión y la mayor generación de subproductos como el material particulado y el carbono negro.

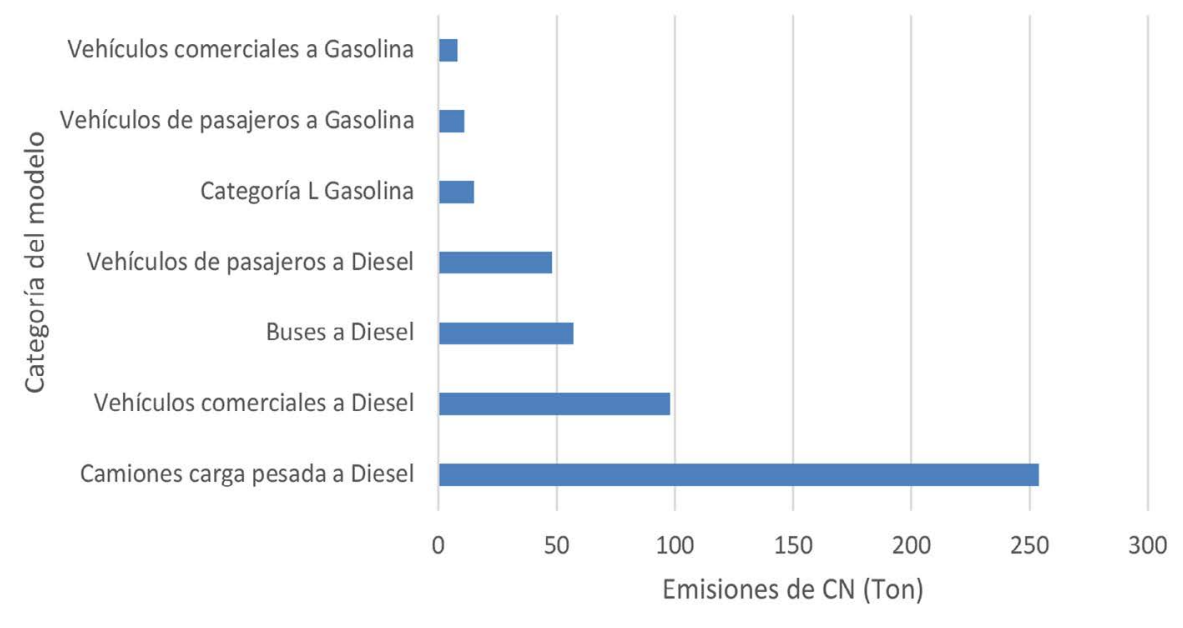

Figura 1. Emisiones de carbono negro distribuidos por categoría de flota vehicular de Costa Rica en 2016. Figure 1. Black carbon emissions distributed by vehicle fleet category in Costa Rica in 2016.

\begin{tabular}{|c|c|c|c|c|c|}
\hline 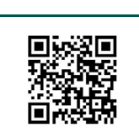 & (c) (1) () (2) () & $\bigotimes_{\text {AMBDETIIIS }}$ & $\frac{2 \%}{2 \% O}$ & $\frac{\text { UNA }}{\frac{\text { WNNERSDAD }}{\text { WNAINAL }}}$ & 166 \\
\hline
\end{tabular}




\section{Revista de CIENCIAS AMBIENTALES Tropical Journal of Environmental Sciences}

Revista de Ciencias Ambientales (Trop J Environ Sci) e-ISSN: 2215-3896

(Enero-Junio, 2022) . Vol 56(1): 157-177 DOI: https://doi.org/10.15359/rca.56-1.8

Open Access: www.revistas.una.ac.cr/ambientales e-mail: revista.ambientales@una.ac.cr Alvarado-Jiménez D., Herrera-Murillo J., Rojas-Marín J.,

La Figura 2 presenta las emisiones por tipo de estándar de vehículo (se incluyen únicamente automotores diésel). Los resultados revelan que un $89.5 \%$ de las emisiones totales de carbono negro de la flotilla vehicular costarricense fue generado por vehículos anteriores al 2005 (previo a Euro 4), los cuales apenas representan el $10.4 \%$ de la flotilla nacional. El $42.4 \%$ de estas fue generado por vehículos con estándar convencional (previo a 1994), cuyo peso porcentual en la flota vehicular es de apenas un $3.4 \%$. Al respecto, Kholod et al. (2016) mostraron resultados similares en sus investigaciones: vehículos pre-euro emitieron el $55 \%$ del total de las emisiones de carbono negro. De igual forma, Cruz-Núñez (2014) y Lau et al. (2015) señalan el impacto de la antigüedad de la flotilla vehicular como un factor determinante en la generación de carbono negro y comprueban la incidencia de las normativas de control de emisiones (Euro) y las tecnologías asociadas a los automotores para el cumplimiento de la reducción directa de las emisiones.

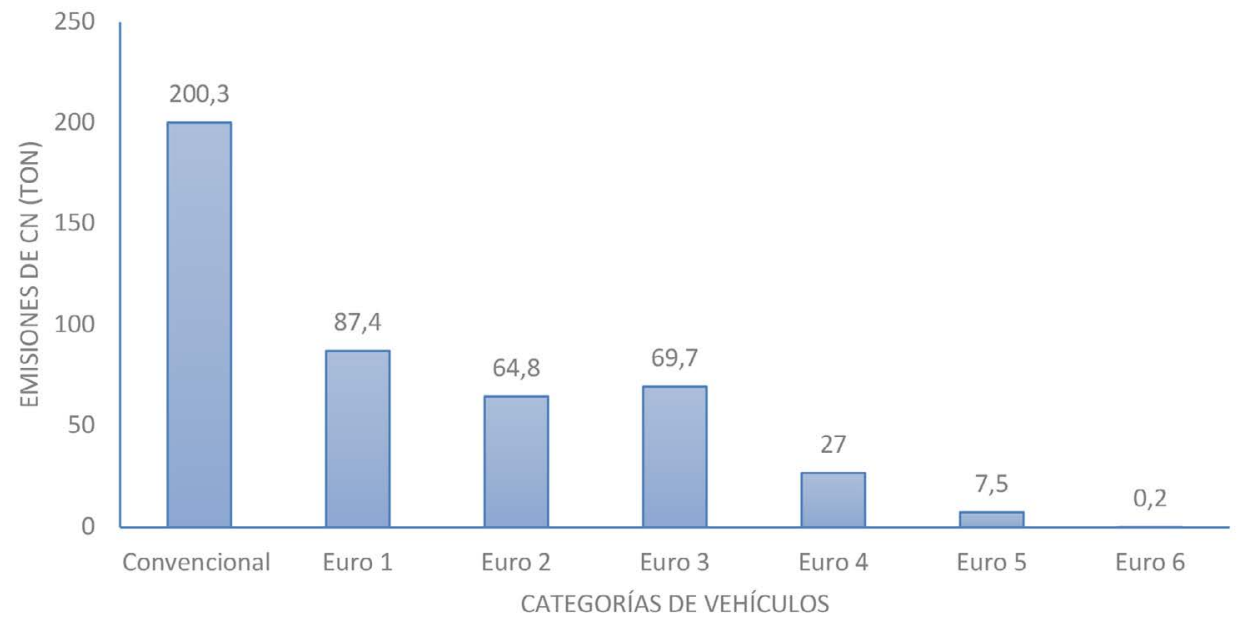

Figura 2. Emisiones de carbono negro $(\mathrm{CN})$ por estándar de vehículo.

Figure 2. Black carbon $(\mathrm{CN})$ emissions by vehicle standard.

El Cuadro 6 presenta las toneladas de $\mathrm{CO}_{2 \mathrm{e}}$ generadas a raíz de las emisiones de carbono negro en dos escenarios distintos: uno conservador, con PCG de 460, y el escenario crítico, con un PCG de 1500.

\begin{tabular}{|c|c|c|c|c|c|}
\hline 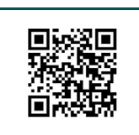 & (c) (i) () (2) & $\Leftrightarrow$ & $\frac{2 \%}{280}$ & 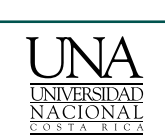 & 167 \\
\hline
\end{tabular}




\section{Revista de CIENCIAS AMBIENTALES Tropical Journal of Environmental Sciences}

Revista de Ciencias Ambientales (Trop J Environ Sci) e-ISSN: 2215-3896

(Enero-Junio, 2022) . Vol 56(1): 157-177 DOI: https://doi.org/10.15359/rca.56-1.8

Open Access: www.revistas.una.ac.cr/ambientales e-mail: revista.ambientales@una.ac.cr Alvarado-Jiménez D., Herrera-Murillo J., Rojas-Marín J., González-Rodríguez M.

Cuadro 6. Emisiones de $\mathrm{CO}_{2 \mathrm{e}}$ resultantes de las emisiones de carbono negro en el sector transporte 2016. Table 6. $\mathrm{CO}_{2}$ e emissions resulting from black carbon emissions in the transport sector 2016.

\begin{tabular}{lccc}
\hline Categoría & $\begin{array}{c}\text { Emisiones de } \mathrm{CO}_{2} \mathrm{e} \\
\text { (ton) PCG 460 }\end{array}$ & $\begin{array}{c}\text { Emisiones de } \mathrm{CO}_{2} \mathrm{e} \\
\text { (ton) } \\
\text { PCG 1500 }\end{array}$ & \% $\mathrm{CO}_{2} \mathrm{e}$ \\
\hline Camiones carga pesada a Diesel & 116081 & 378525 & 53.5 \\
Vehículos comerciales a Diesel & 43862 & 143029 & 20.2 \\
Buses Diesel & 28057 & 91490 & 12.9 \\
Vehículos de pasajeros a Diesel & 22193 & 72367 & 10.2 \\
Categoría L Gasolina & 4880 & 15912 & 2.2 \\
Vehículos de pasajeros a gasolina & 1733 & 5651 & 0.80 \\
Vehículos comerciales a Gasolina & 225.9 & 736.6 & 0.10 \\
\hline Total & 217031 & $\mathbf{7 0 7 7 1 2}$ & \\
\hline
\end{tabular}

En total, se contabilizan entre 217031 y 707712 toneladas de $\mathrm{CO}_{2 \mathrm{e}}$ emitidas por el sector transporte en el país. Esto representa entre un $4 \%$ y un $13 \%$ de la huella de $\mathrm{CO}_{2}$ reportada durante el 2015.

\subsection{Establecimiento de medidas de mitigación y posibles escenarios de reducción}

Con base en la literatura analizada, se muestran algunas de las medidas de reducción más implementadas a nivel global para mitigar las emisiones de carbono negro en la flotilla vehicular.

\subsection{Filtros de partículas}

En términos generales, el objetivo del filtro de partículas diésel (DPF, por sus siglas en inglés: "Diesel Particulate Filter") es evitar la salida a la atmósfera de partículas para después eliminarlas en un proceso de oxidación, conocido como regeneración. El filtro consta de una superficie en forma de canal de abejas hecha de material poroso, con canales alternos que forzosamente direccionan el flujo de los gases de escape a través de ellos (Cortés Duarte, 2016). Los DPF se pueden clasificar, por el principio de funcionamiento, como activos o pasivos. Los pasivos se regeneran en condiciones habituales (temperatura de escape relativamente baja) y los activos, mediante el aumento de la temperatura de escape, lo cual se logra realizando una post-inyección de combustible (Valdepeñas-García, 2018). Con respecto a políticas públicas, no se encontró legislación, planes nacionales u otra documentación para favorecer la implementación de dicha medida.

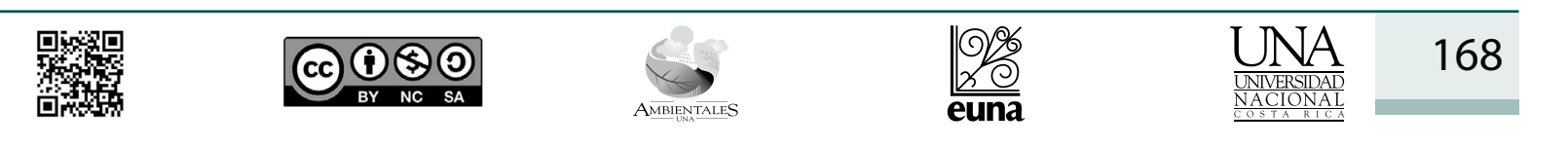




\section{Revista de CIENCIAS AMBIENTALES Tropical Journal of Environmental Sciences}

Revista de Ciencias Ambientales (Trop J Environ Sci) e-ISSN: 2215-3896

(Enero-Junio, 2022) . Vol 56(1): 157-177 DOI: https://doi.org/10.15359/rca.56-1.8

Open Access: www.revistas.una.ac.cr/ambientales e-mail: revista.ambientales@una.ac.cr Alvarado-Jiménez D., Herrera-Murillo J., Rojas-Marín J., González-Rodríguez M.

\subsection{Disminución de azufre en el combustible}

El azufre, además de ser un contaminante importante a nivel ambiental, impide la adopción de tecnologías para el control de la contaminación. Una de las principales problemáticas es que dicho contaminante reduce la eficiencia de conversión del CO, los $\mathrm{HC}$ y el NOx en el catalizador, ya que compite con estos gases por superficie de reacción. Adicionalmente, el contenido de azufre en el combustible contribuye a su envejecimiento, una degradación más seria y una recuperación menos completa (Blumberg et al., 2003). Debido a esto, los combustibles de bajo azufre $(15 \mathrm{ppm})$ generan mayores beneficios al incorporar tecnologías avanzadas de control para vehículos diésel. Los filtros de partículas del diésel pueden usarse con combustibles que no sean de bajo azufre, pero solo alcanzan un $50 \%$ de eficiencia de control, aproximadamente (Comisión Económica para América Latina y el Caribe [CEPAL], 2012).

Por último, en lo que respecta a la vinculación con políticas púbicas, la legislación costarricense establece un máximo de 50 ppm de azufre (Decreto No 36372-MINAET). No obstante, aún no se han implementado regulaciones para el diésel ultra bajo en azufre, pese a que el Reglamento para el control de las emisiones contaminantes producidas por los vehículos automotores con motor de combustión interna $\mathrm{N}^{\circ} 39724$-MOPT-MINAE-S establece que los vehículos a partir del 2021 deben poseer tecnologías para el tratamiento de emisiones de partículas y la utilización de combustibles ultra bajos en azufre es indispensable para asegurar su correcto funcionamiento.

\subsection{Uso de biodiésel}

Dada la relación encontrada en diversos artículos científicos, entre las emisiones de material particulado y los altos niveles de aromáticos y azufre en el combustible (Kholod et al., 2016) se han estudiado combustibles alternativos al diésel que reduzcan su efecto sobre la salud de la población humana. Una de las alternativas es el biodiésel, sea este usado como único combustible o mezclado con el diésel de petróleo.

Al respecto, Magara Gómez (2011), en un estudio donde se realizaron mediciones de las emisiones de carbono negro mezclando biodiésel proveniente de soja y biodiésel proveniente de sebo de carne, mostró que las tasas de emisión de $\mathrm{CN}$ disminuyeron desde un $57 \%$ hasta un $80 \%$ para las mezclas de soja, según la cantidad empleada (mezclas entre el $25 \%$ y 100 \%, respectivamente). De igual forma, para las mezclas de sebo de carne, las disminuciones rondaron entre el $65 \%$ y el $82 \%$. Estas reducciones también son consistentes con las presentadas en el material particulado, donde Hernández Carrillo (2017) indicó una disminución del 50 \% de las emisiones usando una mezcla de $5 \%$ de biodiésel y reportó bajas en las concentraciones de un $20 \%$ y un $50 \%$ para mezclas de $5 \%$ y $10 \%$ de biodiésel, respectivamente.

Los estudios sugieren que la reducción en las emisiones de material particulado se debe al contenido de oxígeno de la mezcla de biodiésel, el cual sustituye al oxígeno del aire en las regiones de mezcla ineficiente del combustible con el aire (Rojas, 2004).

\begin{tabular}{|c|c|c|c|c|c|}
\hline 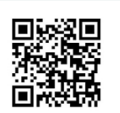 & (c) (i) () & $\underbrace{}_{\text {AMBIFNTIISS }}$ & $\begin{array}{l}\frac{9 \%}{2} \\
\frac{2}{2} \\
\text { euna }\end{array}$ & 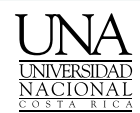 & 169 \\
\hline
\end{tabular}




\section{Revista de CIENCIAS AMBIENTALES Tropical Journal of Environmental Sciences}

Revista de Ciencias Ambientales (Trop J Environ Sci) e-ISSN: 2215-3896

(Enero-Junio, 2022) . Vol 56(1): 157-177 DOI: https://doi.org/10.15359/rca.56-1.8

Open Access: www.revistas.una.ac.cr/ambientales e-mail: revista.ambientales@una.ac.cr Alvarado-Jiménez D., Herrera-Murillo J., Rojas-Marín J., González-Rodríguez M.

En Costa Rica, actualmente, no se comercializa este producto a gran escala; sin embargo, la Refinadora Costarricense de Petróleo, en su Plan de Descarbonización del Sector Transporte Terrestre (2018), plantea introducirlo al país, debido a sus ventajas a nivel ambiental en la reducción de emisiones y a la oportunidad que tiene el país como productor local. No obstante, para lograrlo se deben realizar reformas en la legislación, principalmente en la Ley 6588, que regula la Refinadora Costarricense de Petróleo (RECOPE), para que pueda comercializar este combustible.

\subsection{Vehículos eléctricos}

Los vehículos eléctricos se han posicionado como una alternativa al motor de combustión a nivel global, principalmente por la alta eficiencia que presenta (aproximadamente un $90 \%$, mientras que la de un motor de combustión ronda el 20 \%) (Geraldo Costa, 2019). Este diferencial provoca que la cantidad neta de energía requerida sea menor, lo que implica una reducción del costo energético para los usuarios y una mejora de la intensidad energética de la economía nacional (MINAE, 2019).

Ahora bien, de acuerdo con el Plan Nacional de Transporte Eléctrico 2018-2023, en Costa Rica la participación de la energía eléctrica en el transporte es de solamente el $0.2 \%$. Se espera que la planificación e implementación de políticas orientadas a fortalecer el sector, puedan provocar un aumento al 13.9 \% para el año 2030. Este aumento implicaría una reducción del $27 \%$ en el consumo energético (Ministerio de Ambiente y Energía, 2019).

Sin embargo, a pesar de los beneficios de los vehículos eléctricos, en la actualidad, aún existen importantes brechas en el acceso a estas tecnologías, debido al precio en el mercado; por ejemplo, un autobús de $12 \mathrm{~m}$ puede rondar los 390 mil dólares, mientras que un Euro VI, 200 mil dólares (Restrepo, 2018).

\subsection{Escenarios de reducción teórica de carbono negro}

El Cuadro 7 muestra las medidas anteriormente descritas y los diferentes escenarios de emisión de carbono negro tomando en cuenta su implementación Los resultados señalan que, de no ejecutarse ninguna medida, para el 2025 se presentará un aumento del $66 \%$ de las emisiones de este contaminante y, para el 2030, el aumento corresponderá a un $75 \%$ con respecto al 2016. No obstante, con la aplicación de las medidas presentadas se pueden lograr reducciones significativas.

\begin{tabular}{|c|c|c|}
\hline 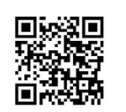 & (c) () () () & 170 \\
\hline
\end{tabular}




\section{Revista de CIENCIAS AMBIENTALES Tropical Journal of Environmental Sciences}

Revista de Ciencias Ambientales (Trop J Environ Sci) e-ISSN: 2215-3896

(Enero-Junio, 2022) . Vol 56(1): 157-177 DOI: https://doi.org/10.15359/rca.56-1.8

Open Access: www.revistas.una.ac.cr/ambientales e-mail: revista.ambientales@una.ac.cr Alvarado-Jiménez D., Herrera-Murillo J., Rojas-Marín J., González-Rodríguez M

Cuadro 7. Potencial de reducción de emisiones de $\mathrm{CN}$ para los escenarios propuestos Table 7. Reduction Potential for $\mathrm{CN}$ emissions for the proposed scenarios

\begin{tabular}{|c|c|c|c|}
\hline Medida & Descripción del alcance del escenario & Reducción & Costo de la tecnología \\
\hline \multirow[t]{3}{*}{$\begin{array}{l}\text { Uso de filtros } \\
\text { antipartículas }\end{array}$} & $\begin{array}{l}\text { Modernización de vehículos diésel carga liviana, } \\
\text { carga pesada y autobuses, con modelo entre } 1995 \text { y } \\
\text { año } 2006 \text { (Euro 3) con filtros antipartículas diésel. }\end{array}$ & & \multirow[t]{3}{*}{$\begin{array}{l}3000-9000 \text { dólares por } \\
\text { unidad }\end{array}$} \\
\hline & $\begin{array}{l}\text { Opción 1: Se propone adaptación de un } 15 \% \text { de las } \\
\text { unidades para el } 2025 .\end{array}$ & 12.6 & \\
\hline & $\begin{array}{l}\text { Opción 2: Se propone adaptación de un } 75 \% \text { de la } \\
\text { flotilla para el } 2030 .\end{array}$ & 62.9 & \\
\hline \multirow{3}{*}{$\begin{array}{l}\text { Uso de combustibles } \\
\text { bajos en azufre }\end{array}$} & Opción 1: & 45.0 & \multirow{3}{*}{$\begin{array}{l}\text { No se comercializa en } \\
\text { el país. Su precio podría } \\
\text { rondar un } 10 \% \text { más del } \\
\text { valor actual }\end{array}$} \\
\hline & $\begin{array}{l}\text { Establecimiento de límite de concentración de } \\
\text { diésel a } 15 \text { ppm. Escenario } 2025 \text {. }\end{array}$ & & \\
\hline & $\begin{array}{l}\text { Opción 2: } \\
\text { Establecimiento de límite de concentración de } \\
\text { diésel a } 15 \text { ppm. Escenario } 2030 \text {. }\end{array}$ & 63.2 & \\
\hline \multirow[t]{2}{*}{ Uso de biodiesel } & $\begin{array}{l}\text { Opción 1: } \\
\text { Utilización de mezcla de diésel con biodiésel a un } \\
5 \% \text { de biodiésel, } 95 \% \text { de diésel en vehículos de } \\
\text { carga liviana, carga pesada y autobuses. Escenario } \\
2025 \text {. }\end{array}$ & 135.8 & \multirow[t]{2}{*}{$\begin{array}{l}\text { No se comercializa en el } \\
\text { país y no está regulada } \\
\text { su venta }\end{array}$} \\
\hline & $\begin{array}{l}\text { Opción 2: Utilización de mezcla de diésel con } \\
\text { biodiésel a un } 5 \% \text { de biodiésel, } 95 \% \text { de diésel } \\
\text { en vehículos de carga liviana y carga pesada y } \\
\text { autobuses. Escenario } 2030 .\end{array}$ & 143.1 & \\
\hline \multirow{3}{*}{$\begin{array}{l}\text { Inclusión de autobuses } \\
\text { eléctricos }\end{array}$} & Opción 1: & 2.1 & \multirow{3}{*}{$\begin{array}{l}390 \text { mil dólares por } \\
\text { unidad }\end{array}$} \\
\hline & $\begin{array}{l}\text { Incorporar dentro de la flota vehicular del país } \\
\text { buses eléctricos para transporte público. Se plantea } \\
\text { para } 2025 \text { que el } 15 \% \text { de la flota sea eléctrica: se } \\
\text { propone que el } 65 \% \text { de los vehículos eléctricos } \\
\text { sustituyan al vehículo Euro } 5 \text { y el } 35 \% \text { restante } \\
\text { corresponda al aumento del parque automotor } \\
\text { Euro } 6 .\end{array}$ & & \\
\hline & $\begin{array}{l}\text { Opción } 2 \text { : } \\
\text { Incorporar dentro de la flota vehicular del país } \\
\text { buses eléctricos para transporte público. Se plantea } \\
\text { para } 2030 \text { que el } 50 \% \text { de la flota sea eléctrica; se } \\
\text { propone que el } 65 \% \text { de los vehículos eléctricos } \\
\text { sustituyan a los vehículos Euro } 6 \text { y el } 35 \% \text { restante } \\
\text { corresponda al aumento del parque automotor. }\end{array}$ & 2.3 & \\
\hline
\end{tabular}

(cc)(3)




\section{Revista de CIENCIAS AMBIENTALES Tropical Journal of Environmental Sciences}

Revista de Ciencias Ambientales (Trop J Environ Sci) e-ISSN: 2215-3896

(Enero-Junio, 2022) . Vol 56(1): 157-177 DOI: https://doi.org/10.15359/rca.56-1.8

Open Access: www.revistas.una.ac.cr/ambientales e-mail: revista.ambientales@una.ac.cr Alvarado-Jiménez D., Herrera-Murillo J., Rojas-Marín J., González-Rodríguez M.

\subsection{Evaluación de medidas de reducción}

El siguiente paso consistió en identificar medidas de reducción para las principales fuentes de carbono negro. En el Cuadro 8 se muestran estas medidas, tomando en cuenta dos escenarios de emisión a futuro y el puntaje obtenido en la evaluación.

Cuadro 8. Evaluación de medidas de reducción de carbono negro.

Table 8. Evaluation of black carbon reduction measures.

\begin{tabular}{|c|c|c|c|c|}
\hline Medida & $\begin{array}{l}\text { Potencial de } \\
\text { reducción }\end{array}$ & $\begin{array}{l}\text { Concordancia con } \\
\text { políticas públicas }\end{array}$ & $\begin{array}{l}\text { Costo de la } \\
\text { tecnología }\end{array}$ & $\begin{array}{l}\text { Puntuación } \\
\text { obtenida }\end{array}$ \\
\hline $\begin{array}{l}\text { Uso de filtros } \\
\text { antipartículas } 2025\end{array}$ & Bajo & Bajo & Alto & 10 \\
\hline $\begin{array}{l}\text { Uso de filtros } \\
\text { antipartículas } 2030\end{array}$ & Medio & Bajo & Alto & 12 \\
\hline $\begin{array}{l}\text { Inclusión de autobuses } \\
\text { eléctricos } 2025\end{array}$ & Bajo & Alto & Alto & 12 \\
\hline $\begin{array}{l}\text { Inclusión de autobuses } \\
\text { eléctricos } 2030\end{array}$ & Bajo & Alto & Alto & 18 \\
\hline Uso de biodiésel 2025 & Alto & Alto & Alto & 18 \\
\hline Uso de biodiésel 2030 & Alto & Alto & Alto & 18 \\
\hline $\begin{array}{l}\text { Uso de combustibles bajos } \\
\text { en azufre } 2025\end{array}$ & Medio & Medio & Alto & 18 \\
\hline $\begin{array}{l}\text { Uso de combustibles bajos } \\
\text { en azufre } 2030\end{array}$ & Medio & Medio & Alto & 18 \\
\hline
\end{tabular}

Los resultados muestran que las medidas de uso de combustibles bajos en azufre, uso de biodiésel e inclusión de buses eléctricos para el escenario 2030 son las más factibles para poner en práctica en el país; contrario a esto, la medida peor calificada es el uso de filtros DAF para el escenario 2025.

Además, se evidencia que las medidas relacionadas con la calidad y el tipo de combustibles cuentan con una mayor factibilidad de implementación. Lo anterior porque pueden ser aplicables a corto plazo, pues no necesitan ninguna modificación del vehículo, conllevan beneficios en el rendimiento del combustible, e incluso, en el caso del combustible bajo en azufre, implica mejoras en el funcionamiento y alargamiento de la vida útil del motor. Además de esto, son medidas que se han venido trabajado dentro del marco de las políticas públicas (como el Plan de Descarbonización del Sector Transporte Terrestre (2018) y el Decreto No 36372-MINAET), en las que se evidencia el interés del mercado por la calidad del diésel.

En cuanto a la implementación de filtros, su baja puntuación se debe principalmente al costo de la tecnología y de su mantenimiento; por la inexistencia de precedentes en políticas públicas y, finalmente, por el bajo impacto en las reducciones de las emisiones de carbono

\begin{tabular}{|c|c|c|c|c|c|}
\hline 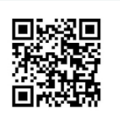 & (c) (i) (9) & $\overbrace{\text { AMBENTALS }}^{\infty}$ & $\frac{10 \%}{\text { euna }}$ & $\frac{\text { UNA }}{\frac{\text { UNIIERSIDAD }}{\text { UASIONAL }}}$ & 172 \\
\hline
\end{tabular}




\section{Revista de CIENCIAS AMBIENTALES Tropical Journal of Environmental Sciences}

Revista de Ciencias Ambientales (Trop J Environ Sci) e-ISSN: 2215-3896

(Enero-Junio, 2022) . Vol 56(1): 157-177 DOI: https://doi.org/10.15359/rca.56-1.8

Open Access: www.revistas.una.ac.cr/ambientales e-mail: revista.ambientales@una.ac.cr Alvarado-Jiménez D., Herrera-Murillo J., Rojas-Marín J., González-Rodríguez M.

negro para los sectores en estudio (menos del $10 \%$, incluso previendo la incorporación del $75 \%$ de la flotilla).

Estos resultados son respaldados por los obtenidos por Lau et al. (2015), quienes señalan que esta medida es poco eficiente para controlar las emisiones, debido a la falta de controles para asegurar un correcto mantenimiento de los filtros.

Por último, las medidas de reducción referentes a incorporar vehículos eléctricos son factibles a largo plazo, más no a corto plazo, especialmente por el costo actual de las unidades de transporte y porque, tomando en cuenta la situación actual del país y la fluctuación en los precios del petróleo, la importación y sustitución de los autobuses no será tan rentable como se estimaba.

\section{Conclusiones}

El carbono negro es un agente de calentamiento global que empieza a tener relevancia por las implicaciones a corto plazo que podría conllevar la reducción de sus emisiones en materia de cambio climático. En la presente investigación, se evidencia la importancia de este contaminante, ya que su aporte es de entre un $4 \%$ y un $13 \%$ más para el sector transporte durante el 2016, en comparación con el inventario de GEI del año 2015 (MINAE, 2019).

Este estudio destaca que la mayor parte de las emisiones se producen por vehículos de carga y autobuses, principalmente aquellos con más de 25 años de antigüedad, en el caso de camiones, por lo que es importante incentivar políticas públicas que no solo regulen la importación de vehículos sino también la renovación de la flotilla vehicular existente.

A nivel internacional, ya se ha venido trabajando en medidas puntuales para la reducción de carbono negro, entre las cuales se destacan el uso de combustibles bajos en emisiones, vehículos eléctricos y mejoras tecnológicas en el sistema del motor para reducir las emisiones de escape. Los hallazgos de esta investigación señalan que, dado el contexto económico y político del país, las medidas que tienen una mayor factibilidad son las relacionadas con la utilización de combustibles con bajas emisiones, como el biodiésel y el diésel bajo en azufre, ya que alcanzan a toda la flotilla vehicular y los propietarios no necesitarían recurrir a modificaciones en sus vehículos. En contraposición, incorporar vehículos eléctricos y remodelar vehículos antiguos con sistemas de tratamiento en el escape son medidas poco rentables, por lo menos a corto plazo $y$, por ende, con una baja aceptación social.

Es necesario destacar que las emisiones estimadas durante este estudio podrían verse afectadas por las limitaciones en cuanto a la información existente en el país. Entre ellos podemos indicar que, a la fecha, no se tiene documentado el estándar de emisiones de cada vehículo, ni el kilometraje; además, existen inconsistencias en cuanto al peso y cilindraje de un porcentaje importante de la flotilla y no se cuenta con estadísticas de las velocidades promedio en zonas rurales, urbanas y autopistas. Todas estas estimaciones aportan incertidumbre en las emisiones reportadas en este estudio.

\begin{tabular}{|c|c|c|c|c|}
\hline 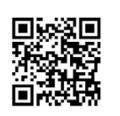 & (c) (1) $\$(0)$ & $\overbrace{\text { AMBIFINIIIS }}$ & $\frac{10 \%}{\text { euna }}$ & 173 \\
\hline
\end{tabular}




\section{Revista de CIENCIAS AMBIENTALES Tropical Journal of Environmental Sciences}

Revista de Ciencias Ambientales (Trop J Environ Sci) e-ISSN: 2215-3896

(Enero-Junio, 2022) . Vol 56(1): 157-177 DOI: https://doi.org/10.15359/rca.56-1.8

Open Access: www.revistas.una.ac.cr/ambientales e-mail: revista.ambientales@una.ac.cr Alvarado-Jiménez D., Herrera-Murillo J., Rojas-Marín J., González-Rodríguez M.

\section{5. Ética y conflicto de intereses}

Las personas autoras declaran que han cumplido totalmente con todos los requisitos éticos y legales pertinentes, tanto durante el estudio como en la producción del manuscrito; que no hay conflictos de intereses de ningún tipo; que todas las fuentes financieras se mencionan completa y claramente en la sección de agradecimientos, y que están totalmente de acuerdo con la versión final editada del artículo.

\section{Referencias}

Agencia Europea de Medio Ambiente (AEMA). (2021). COPERT 5 Manual. https://copert.emisia.com/

Badii, M. H., Guillén, A., Lugo, O., \& Sánchez, H. J. (2015). Aspectos del Calentamiento. Daena: International Journal of Good Conscience, 10(2), 175-195. http://www.spentamexico.org/ v10-n2/A11.10(2)175-195.pdf

Blumberg, K., Walsh, C., \& Pera, K. (2003). Gasolina y diésel de bajo azufre: La clave para disminuir las emisiones vehiculares. https://theicct.org/sites/default/files/Bajo_Azufre_ICCT_2003.pdf

Ceballos Marcillo, J. J. (2016). Simulation of Fuel Consumption and Emissions of the Public Transportation Fleet in Ambato (Ecuador) With Copert 4. [Tesis de máster, Universidad de Valladolid]. https://doi.org/10.13140/RG.2.2.32397.54247

Chow, J. C., Watson, J. G., Lowenthal, D. H., Antony Chen, L.-W., \& Motallebi, N. (2011). PM2.5 source profiles for black and organic carbon emission inventories. Atmospheric Environment, 45(31), 5407-5414. https://doi.org/10.1016/j.atmosenv.2011.07.011

Comisión Económica para América Latina y el Caribe (2012). Análisis de la reducción del azufre en el combustible diésel en El Salvador, Guatemala y Nicaragua. https://www.cepal.org/ es/publicaciones/26095-analisis-la-reduccion-azufre-combustible-diesel-salvador-guatemala-honduras

Cortés Duarte, M. C. (2016). Eficiencia de remoción de material particulado usando filtros de partículas en vehículos diésel del Sistema Integrado de Transporte Público de Bogotá DC [Tesis de Maestría, Universidad Nacional de Colombia]. Repositorio UNAL. https://repositorio. unal.edu.co/handle/unal/57778

Geraldo Costa, J. E. (2019). Mass introduction of electric passenger vehicles in Brazil: Impact assessment on energy use, climate mitigation and on charging infrastructure needs for several case studies. [Tesis de Doctorado, Universidade Nova]. Repositório Universidade Nova. https:// run.unl.pt/handle/10362/83963

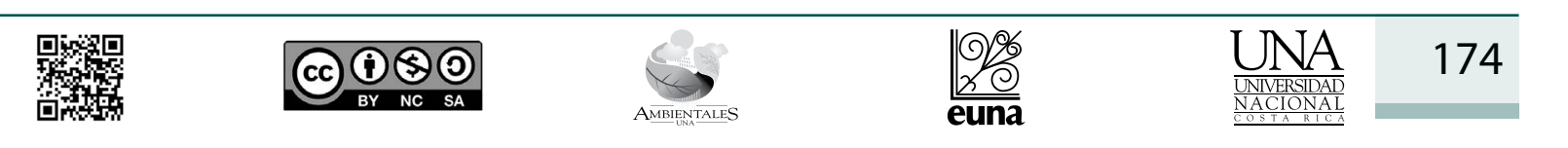




\section{Revista de CIENCIAS AMBIENTALES Tropical Journal of Environmental Sciences}

Revista de Ciencias Ambientales (Trop J Environ Sci) e-ISSN: 2215-3896 (Enero-Junio, 2022) . Vol 56(1): 157-177 DOI: https://doi.org/10.15359/rca.56-1.8 Open Access: www.revistas.una.ac.cr/ambientales e-mail: revista.ambientales@una.ac.cr Alvarado-Jiménez D., Herrera-Murillo J., Rojas-Marín J., González-Rodríguez M.

Cruz-Núñez, X. (2014). An approach to a black carbon emission inventory for Mexico by two methods. Science of The Total Environment, 479-480, 181-188. https://doi.org/10.1016/j. scitotenv.2014.01.064

Fugless, J. S., Shine, K. P., Berntsen, T., Cook, J., Lee, D. S., Stenke, A., \& Waitz, I. A. (2010). Transport impacts on atmosphere and climate: Metrics. Atmospheric Environment, 44(37), 4648-4677. https://doi.org/10.1016/j.atmosenv.2009.04.044

Gallego Picó, A., González Fernández, I., Sánchez Gimeno, B., Fernández Hernaldo, P., Garcimuño Martínez, R., Bravo Yagüe, J. C., Pradana Pérez, J. A., Navarro Pascual, R. García Mayor, A., \& Durand Alegría, J. S. (2012). Contaminación atmosférica. Universidad Nacional de Educación a Distancia (UNED). http://site.ebrary.com/lib/unacrsp/reader. action? docID $=10646498 \&$ ppg $=57$

Gladstein, Neandross \& Associates. (2014). Dumping Dirty Diesels in Latin America: Reducing Black Carbon and Air Pollution from Diesel Engines in Latin American Countries. 48. https:// www.nrdc.org/resources/dumping-dirty-diesels-latin-america

Gobierno de Costa Rica, Ministerio de Ambiente y Energía (2015). Contribución Prevista y Determinada a Nivel Nacional de Costa Rica. http://www4.unfccc.int/ndcregistry/PublishedDocuments/Costa\%20Rica\%20First/INDC\%20Costa\%20Rica\%20Version\%202\%200\%20 final\%20ES.pdf

Grupo Intergubernamental sobre el Cambio Climático (2007). Gases de efecto invernadero - CIE WGI Resumen Técnico. (Fourth Assessment Report, Climate Change) IPCC. https://www. ipcc.ch/publications_and_data/ar4/wg1/es/tssts-2-1.html

Grupo Intergubernamental sobre el Cambio Climático (IPCC). (2014). Cambio climático 2014: Mitigación del Cambio Climático: Resumen para responsables de políticas y resumen técnico. https://www.ipcc.ch/site/assets/uploads/2018/03/WGIIIAR5_SPM_TS_Volume_es-1.pdf

Hernández Carrillo, A. (2017). Evaluación de la cinética de combustión del aceite, biodiésel y mezclas de Jatropha y sus emisiones de material particulado en motores de combustión interna [Tesis doctoral, Universidad Nacional de Colombia]. Repositorio Institucional UNAL. https://repositorio.unal.edu.co/handle/unal/59899

Kholod, N., Evans, M., Gusev, E., Yu, S., Malyshev, V., Tretyakova, S., \& Barinov, A. (2016). A methodology for calculating transport emissions in cities with limited traffic data: Case study of diesel particulates and black carbon emissions in Murmansk. Science of The Total Environment, 547, 305-313. https://doi.org/10.1016/j.scitotenv.2015.12.151

\begin{tabular}{|c|c|c|c|c|c|}
\hline 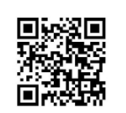 & (c) (i) (\$) & $\underset{\text { AMEIENTALS }}{3}$ & $\frac{1 \%}{2 \%}$ & $\frac{\text { UNA }}{\frac{\text { UNIEESIIDAD }}{\text { NACRIONAL }}}$ & 175 \\
\hline
\end{tabular}




\section{Revista de CIENCIAS AMBIENTALES Tropical Journal of Environmental Sciences}

Revista de Ciencias Ambientales (Trop J Environ Sci) e-ISSN: 2215-3896

(Enero-Junio, 2022) . Vol 56(1): 157-177 DOI: https://doi.org/10.15359/rca.56-1.8

Open Access: www.revistas.una.ac.cr/ambientales e-mail: revista.ambientales@una.ac.cr Alvarado-Jiménez D., Herrera-Murillo J., Rojas-Marín J., González-Rodríguez M.

IMN. (2017). Datos meteorológicos promedios mensuales obtenidos para las estaciones de monitoreo en Costa Rica, año 2016. Instituto Meteorológico Nacional. Ministerio de Ambiente y Energía. San José, Costa Rica.

Lau, C. F., Rakowska, A., Townsend, T., Brimblecombe, P., Chan, T. L., Yam, Y. S., Močnik, G., \& Ning, Z. (2015). Evaluation of diesel fleet emissions and control policies from plume chasing measurements of on-road vehicles. Atmospheric Environment, 122, 171-182. https://doi.org/10.1016/j.atmosenv.2015.09.048

Magara-Gómez, K. T. (2011). Impact of fossil fuel alternatives on internal combustion engine emissions [Tesis doctoral, University of Wisconsin-Madison]. ProQuest. https://search. proquest.com/openview/26bd37aebc33d54b24eb8d3096cef879/1?pq-origsite $=$ gscholar\&c$\mathrm{bl}=18750 \&$ diss $=\mathrm{y}$

MINAE (2019). Costa Rica 2019: Inventario Nacional de gases de efecto invernadero y absorción de carbono 2015. MINAE

Ministerio de Ambiente y Energía. (2019). Plan nacional de transporte eléctrico 2018-2030. MINAE DGM, SEPSE. https://sepse.go.cr/documentos/PlanTranspElect.pdf

Organización de las Naciones Unidas (1998). Protocolo de Kyoto de la Convención Marco de las Naciones Unidas sobre el cambio climático. https://unfccc.int/resource/docs/convkp/kpspan.pdf

Organización Mundial de la Salud. (2016). Calidad del aire ambiente (exterior) y salud. http:// www.who.int/mediacentre/factsheets/fs313/es

Ortúzar, F., \& Tornel, C. (2016). Controlando los contaminantes climáticos de vida corta: una oportunidad para mejora la calidad del aire y mitigar el cambio climático: el caso de Brasil, Chile y México. Asociación Interamericana para la Defensa del Ambiente (AIDA) e Instituto de Energia e Meio Ambiente (IEMA). https://aida-americas.org/es/controlando-los-contaminantes-clim-ticos-de-vida-corta-una-oportunidad-para-mejorar-la-calidad-del

UNEP \& WMO (2011). Integrated assessment of black carbon and tropospheric ozone: Summary for Decisión Makers. https://wedocs.unep.org/20.500.11822/8028

Programa Estado de la Nación. (2016). Vigésimo segundo informe: Estado de la Nación en Desarrollo Humano Sostenible (22. ${ }^{a}$ ed.). PEN. http://www.estadonacion.or.cr/estado/Publicaciones/Estado_Nacion/22/index.html

\begin{tabular}{|c|c|c|}
\hline 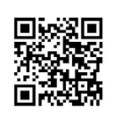 & (c) () & 176 \\
\hline
\end{tabular}




\section{Revista de CIENCIAS AMBIENTALES Tropical Journal of Environmental Sciences}

Revista de Ciencias Ambientales (Trop J Environ Sci) e-ISSN: 2215-3896

(Enero-Junio, 2022) . Vol 56(1): 157-177 DOI: https://doi.org/10.15359/rca.56-1.8 Open Access: www.revistas.una.ac.cr/ambientales e-mail: revista.ambientales@una.ac.cr Alvarado-Jiménez D., Herrera-Murillo J., Rojas-Marín J., González-Rodríguez M.

Restrepo, P. (2018). Metodología para la implementación de buses eléctricos con baterías de litio-titanato en la ruta circular sur 302 de Medellín Colombia [Tesis de máster, Universidad Pontificia Bolivariana]. Repositorio UPB https://repository.upb.edu.co/bitstream/ handle/20.500.11912/4378/Metodolog\%C3\%ADa\%20para\%20la\%20implementaci\%C3\%B3n\%20de\%20buses\%20el\%C3\%A9ctricos\%20con\%20bater\%C3\%ADas\%20litio-litanato\%20en\%20la\%20ruta.pdf? sequence=1\&isAllowed $=\mathrm{y}$

Rojas, N. Y. (2004). Revisión de las emisiones de material particulado por la combustión de diésel y biodiesel. Revista de Ingeniería, 20, 58-68. https://doi.org/10.16924/revinge.20.7

Sánchez Hernández, L. (2018). Estado de la Nación: Diagnóstico sobre la situación del transporte y la movilidad en Costa Rica. Defensoría de los Habitantes; Consejo Nacional de Rectores. https://repositorio.conare.ac.cr/handle/20.500.12337/2962

Sims, R., Gorsevski, V., \& Anenberg S. (2015). Black Carbon Mitigation and the Role of the Global Environment Facility: A STAP Advisory Document. Global Environment Facility.

Stjern, C. W., Samset, B. H., Myhre, G., Forster, P. M., Hodnebrog, O., Andrews, T., Boucher, O., Faluvegi, G., Iversen, T., Kasoar, M., Kharin, V., Kirkevåg, A., Lamarque, J., Olivié, D., Richardson, T., Shawki, D., Shindell, D., Smith, C., Takemura, T., \& Voulgarakis, A. (2017). Rapid adjustments cause weak surface temperature response to increased black carbon concentrations. Journal of Geophysical Research Atmosphere, 122(21), 11462-11481. https://doi.org/10.1002/2017JD027326

UN Environment Programme. (2020). Black Carbon. Climate \& Clean Air Coalition. https://www.ccacoalition.org/en/slcps/black-carbon

Valdepeñas-García Moreno, J. (2018). Caracterización de hollín implicaciones en la reactividad en DPF [Doctoral, Universidad de Castilla de la Mancha]. Repositorio RUIdeRA. https://ruidera.uclm.es/xmlui/handle/10578/18559

\begin{tabular}{|c|c|c|c|c|c|}
\hline 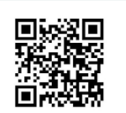 & (c) (i) (9) & $\overbrace{\text { AMBENTALS }}^{\infty}$ & $\frac{O \%}{2)}$ & $\frac{\text { UNA }}{\frac{\text { UNIIERSIDAD }}{\text { UASIONAL }}}$ & 177 \\
\hline
\end{tabular}

